\section{Cardiac involvement in the long-term implications of COVID-19}

\section{Benjamin A. Satterfield([D, Deepak L. Bhatt(1) and Bernard J. Gersh (D)}

Abstract | Throughout 2021, the medical and scientific communities have focused on managing the acute morbidity and mortality caused by the coronavirus disease 2019 (COVID-19) pandemic. With the approval of multiple vaccines, there is a light at the end of this dark tunnel and an opportunity to focus on the future, including managing the long-term sequelae in patients who have survived acute COVID-19. In this Perspectives article, we highlight what is known about the cardiovascular sequelae in survivors of COVID-19 and discuss important questions that need to be addressed in prospective studies to understand and mitigate these lasting cardiovascular consequences, including in post-acute COVID-19 syndrome. To provide the greatest benefit to these survivors, prospective studies should begin now, with resources made available to monitor and study this population in the coming years.

The coronavirus disease 2019 (COVID-19) pandemic is caused by severe acute respiratory syndrome coronavirus 2 (SARS-CoV-2) and is associated with various disease manifestations across multiple organ systems, presenting with a spectrum of disease severity that is only partially explained by age, sex and comorbidities ${ }^{1}$. Of note, the manifestations of acute cardiovascular injury associated with SARS-CoV-2 infection are diverse, including acute myocardial infarction, myocarditis, stress cardiomyopathy, pericarditis, arrhythmias, multisystem inflammatory syndrome in both adults (MIS-A) and children (MIS-C), stroke, macrothrombotic disease including arterial and venous thromboembolism, microthrombotic disease and bleeding diathesis $^{2-4}$ (FIG. 1). The pathogenic mechanisms underlying these clinical manifestations are not well understood and are likely to be multifactorial, including primary causes such as elevated local (endotheliitis) and systemic (cytokine storm) inflammation, resulting in coagulopathy, myocardial infarction, stroke, MIS-A, MIS-C and arrhythmias, direct viral cytopathic effects possibly resulting in myocarditis, and autoantibodies ${ }^{5-7}$. In addition, hypoxaemia can occur secondary to lung injury, and myocardial oedema can develop secondary to the inflammatory states mentioned above $e^{5-7}$.

Many studies have documented substantial increases in serum troponin levels as a manifestation of acute myocardial injury and, in general, these biomarkers have been shown to be correlated with the severity of acute illness ${ }^{2-4,8,9}$ as well as SARS-CoV-2 viraemia ${ }^{10}$. However, among these patients, the degree of myocardial injury observed is inconsistent and the mechanisms are often unclear; whether the degree of troponin level elevation is simply reflective of more severe systemic illness or whether it is a causal factor of worsened prognosis has not yet been determined.

After acute illness, the long-term health implications in survivors of COVID-19 are uncertain, but evidence suggests that they might include cardiovascular, pulmonary, neurological and mental health complications as well as other less understood but debilitating symptoms and features of COVID-19 'long-hauler' syndrome (reviewed elsewhere ${ }^{11}$ ). The rate of these complications is high but the true prevalence is uncertain. Groups such as the Electronic Medical Records and Genomics (eMERGE) consortium are developing electronic phenotyping algorithms to assist researchers in rapidly identifying from the electronic health record the patients with COVID-19 who developed sequelae of the cardiovascular and other organ systems ${ }^{12}$. The high rate of these long-term complications potentially represents a substantial public health burden and well-designed studies are needed to follow-up individuals with post-acute COVID-19 syndrome, which should begin as soon as possible, with several already under way.

The disruption caused by the COVID-19 pandemic led to numerous clinical trials being delayed, cancelled or altered, which, in some cases, has resulted in the deployment of adaptive trial designs to meet the unique circumstances of the pandemic ${ }^{13}$. These trial designs have ultimately improved how clinical trials are performed, including the use of remote consent, telemedicine monitoring and novel trial protocols ${ }^{11,14,15}$. Effects on the 892 cardiovascular trials that were under way before the COVID-19 pandemic have been discussed previously ${ }^{16}$. More than 100 trials relating to COVID-19 with cardiovascular implications have been registered, including registries examining myocarditis, heart failure (HF), venous thromboembolism and general cardiac complications; additionally, there are 19 trials examining imaging, biomarkers and the natural history of cardiac sequelae as well as dozens of other trials on various cardiovascular medications, including the anti-inflammatory agent colchicine, inhibitors of the renin-angiotensinaldosterone system (RAAS), antiplatelet and anticoagulant agents, lipid-lowering therapies, and other drugs for pulmonary hypertension and HF (TABLE 1). Many of these trials were small, had neutral outcomes and were largely underpowered. A few large trials with neutral outcomes included those that assessed the efficacy of ivermectin ${ }^{17}$ and systemic anticoagulation ${ }^{18}$. Early in the pandemic, a theoretical concern arose that RAAS inhibition might worsen COVID-19, but subsequent studies have shown that this fear was unfounded and that removal of RAAS inhibition in patients with COVID-19 already receiving those medications is actually harmful ${ }^{19}$. For all clinical trials during this time, researchers have had to account for elevated rates of hospitalization and death, including a competing risk of death that can affect power calculations as well as under-reporting of events ${ }^{20}$. 
In this Perspectives article, we highlight what is known about cardiovascular sequelae in survivors of COVID-19 and discuss important questions that need to be addressed in prospective studies to understand and mitigate these lasting cardiovascular consequences, including post-acute COVID-19 syndrome.

\section{Cardiovascular complications in post-acute COVID-19}

COVID-19 presents with a spectrum of severity. Throughout this manuscript, we discuss four degrees of severity, as typically used in the medical literature: asymptomatic, mild (having symptoms but not requiring hospitalization), moderate (requiring hospitalization but not intensive care) and severe (requiring intensive care). Some patients recover quickly, whereas others have a more prolonged course to recovery owing to lasting sequelae or the development of persistent symptoms (FIG. 2). When examining cardiovascular consequences in survivors of acute COVID-19, classifying them into four non-exclusive categories might be helpful.

- Persistent symptoms in patients who initially presented with asymptomatic, mild or moderate acute COVID-19 ('long-hauler' COVID-19)

- Evidence of cardiac injury (abnormal cardiac MRI) in patients (including athletes) who had only mild COVID-19

- Cardiovascular complications in patients with moderate-to-severe COVID-19 and evidence of cardiac injury (elevated troponin levels and/or reduced left ventricular ejection fraction)

- 'Collateral damage' in patients owing to delays in presentation to hospital and treatment of acute coronary syndromes, stroke and other cardiovascular disorders

\section{Long-hauler COVID-19}

In a similar manner to survivors of other betacoronavirus infections, such as SARS-CoV and Middle East respiratory syndrome coronavirus (MERS-CoV), a variety of persistent symptoms have been described in survivors of SARS-CoV-2 infection $^{21-27}$. Infectious virus can be isolated from patients up to 3 weeks after the development of acute symptoms, but the presence of persistent symptoms after this time is not thought to be caused by acute viral infection ${ }^{11,28,29}$. These persistent symptoms can be classified as cardiopulmonary (chest pain, palpitations, tachycardia and dyspnoea), neurological (neuropathy, myelopathy,

Stroke
- Hypercoagulability
- Inflammatory milieu
- Bleeding diathesis
mia
icular fibrillation,
cular tachycardia, atrial
ation or flutter, or heart
ine hypoxaemia,
term consequences not
very common post-acute
D-19 symptom

Myocardial infarction

- Secondary to inflammtion-

related plaque destabilization

(type 1) and increased

metabolic demand with

hypoxaemia (type 2)

- Many cases had late

presentation to hospital and

delayed treatment, leading to

less-than-ideal recovery and

probably worse long-term

consequences

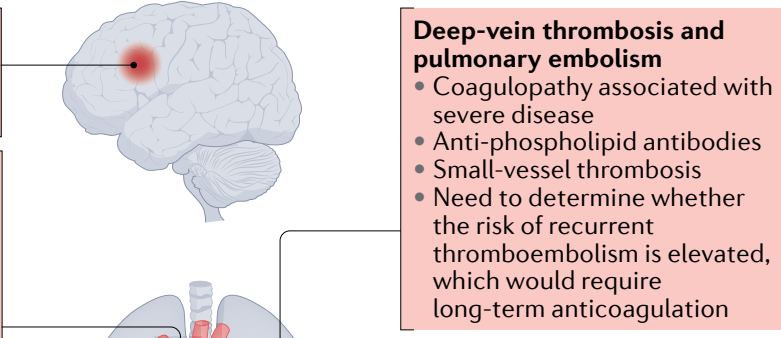

Left and/or right ventricular systolic dysfunction

- Can occur due to myocarditis, stress-induced cardiomyopathy or myocardial infarction - Often transient but time to normalization and long-term consequences not yet well defined

Pericarditis

- Might or might not be associated with elevated troponin levels

- Uncertain whether the risk of complications is increased compared with non-COVID-19related pericarditis

Fig. 1 | Acute cardiovascular complications secondary to COVID-19. Acute coronavirus disease 2019 (COVID-19) can lead to various cardiovascular complications, including arrhythmia, myocardial infarction, deep-vein thrombosis and pulmonary embolism, left and/or right ventricular systolic dysfunction, pericarditis and stroke. These complications can have profound long-term implications for the health of survivors of COVID-19.

cognitive disturbance, dysautonomia, headaches, dysgeusia, and anosmia or parosmia), mental health (depression, anxiety, post-intensive care unit (ICU) syndrome and sleep disturbance) or non-specific (fatigue, joint pain and muscle weakness $)^{30-34}$. This constellation of symptoms falls under the umbrella of COVID-19 long-hauler syndrome.

The aetiology is poorly understood, the morbidity is substantial and the response to multidisciplinary rehabilitation is inconsistent. Whether these symptoms differ between those who initially presented with mild illness and those with moderate or severe illness is uncertain.

Dyspnoea is the most frequent persistent symptom reported and is likely to be multifactorial in aetiology $y^{33-36}$. Although we are focusing on cardiovascular complications, the effect of COVID-19 on respiratory function certainly contributes to the dyspnoea observed in these patients. Chest pain is the most common persistent cardiovascular symptom among patients surviving moderate-to-severe COVID-19; one study reported chest pain in $18 \%$ of survivors at 30 days and in $13 \%$ at 60 days $^{34}$, whereas another study found chest pain in $21 \%$ of survivors at 60 days $^{33}$. An additional study with longer follow-up found chest pain in $5 \%$ of survivors at 6 months ${ }^{35}$. Palpitations were also common, being present in $9 \%$ of survivors at 30 days $^{34}, 14 \%$ at 60 days $^{34}$ and $9 \%$ at 6 months ${ }^{35}$.

A variety of neurological, cardiac, pulmonary and other post-acute COVID-19 symptoms might have a theoretical aetiology in a persistently elevated inflammatory state, especially in those who developed cytokine storm or MIS-A or MIS-C during the acute phase of illness ${ }^{37}$.

Arrhythmias. The increased frequency of heart palpitations has raised a question about arrhythmias in survivors of COVID-19. Both bradyarrhythmias (including high-grade atrioventricular block) and tachyarrhythmias (including atrial fibrillation or flutter, ventricular tachycardia, and ventricular fibrillation) have been reported in acute COVID-19 $\left(\mathrm{REFS}^{38,39}\right.$ ); however, these represent acute arrhythmias in the setting of acute illness and the chronicity of these new arrhythmias is uncertain. Acute arrhythmias were more common in patients with COVID-19 admitted to the ICU than in those admitted to general medical units, although the rates reported in the literature have varied from $18 \%$ to $44 \%{ }^{40-42}$. New electrocardiographic changes and arrhythmias were detected in up to one-third of survivors of non-severe COVID-19 by systematic screening ${ }^{43}$. 


\section{PERSPECTIVES}

Table 1 | Clinical registries and trials studying COVID-19 with cardiovascular implications

Registry, trial or drug name

NCT identifier

Registries

Hospital Registry of Acute Myocarditis: Evolution of the Proportion of Positive SARS-COV-2

NCT04375748

(COVID19) Cases

COLUMBIA CARDS: COvid-19 LongitUdinal Multiethnic Biolmaging Assessment of CARDiovascular Sequelae Registry

CAPACITY-COVID: Cardiac complicAtions in Patients With SARS Corona vlrus 2 (COVID-19) regisTrY

PCHF-COVICAV: COVID-19 in Hospitalized Patients With Preexisting CArdioVascular Diseases and/or Cardiac Involvement and/or Cardiovascular Risk Factors: The Global PCHF-COVICAV Registry

CORONA-VTE NET: COVID-19 Registry to Assess Frequency, Risk Factors, Management, and

Outcomes of Arterial and Venous Thromboembolic Complications

Trials examining natural history, imaging or biomarkers, with an emphasis on cardiac sequelae

Sequelae of Sars-CoV-2 Infections

The Role of Adaptive Immunity in COVID-19 Associated Myocardial Injury

NCT04661657

NCT04325412

NCT04390555

NCT04535128

Right Ventricular Dysfunction in Ventilated Patients With COVID-19

Descriptive and Retrospective Analysis of Acute Myocarditis Associated With Pandemic COVID-19 in Children

Myocardial Injury and Quality of Life After COVID-19

NCT04794062

Prevalence of Perimyocarditis After Covid-19 Vaccine

NCT04865900

Myocardial Involvement of Severe Acute Respiratory Syndrome-Cov-2 (Covid19) Infected Patients

Cardiac Dysfunction in Critically Ill Patients With COVID-19

NCT04358952

Myocardial Injury and Major Adverse Outcomes in Patients With COVID-19

NCT04524234

NCT04397939

Cardiac Injury in COVID-19: a Pathology Study

Multimodal IMAgery Characterization of Cardiac Damage and Severity After COVID-19 Infection

NCT04367792

MYocardial DOmmages Related to COVID-19

NCT04753762

Acute Cardiovascular Events Triggered by COVID-19-Related Stress

NCT04498065

Cardiovascular Complications and COVID-19 (CovCardioVasc-Study)

NCT04368637

Cardiac COVID-19 Health Care Workers

NCT04335162

Mechanical Complications of Acute Myocardial Infarction During COVID-19 Pandemics

NCT04413071

NCT04813692

EPIdemiological Approaches to the Cardiovascular Consequences of COVID-19

NCT04498091

Prevalence of Myocardial Scars on CMR After COVID-19 Infection

NCT04636320

One-year Cardiac Follow-up of Patients With COVID-19 Pneumonia

NCT04501822

Cardiac anti-inflammatory therapy

Colchicine

NCT04416334, NCT04375202, NCT04350320, NCT04724629, NCT04367168, NCT04360980, NCT04359095, NCT04667780, NCT04492358, NCT04324463, NCT04381936, NCT04328480

\section{Renin-angiotensin-aldosterone system inhibition}

Ramipril

Telmisartan

Losartan

Candesartan

NCT04366050

NCT04466241, NCT04359953, NCT04510662, NCT04355936

NCT04643691, NCT04328012

NCT04351724

Spironolactone

NCT04643691

Angiotensin 1-7

NCT04605887, NCT04633772

Antiplatelet or anticoagulant therapy

Aspirin

NCT04333407, NCT04365309,

NCT04324463, NCT04381936

Clopidogrel

NCT04333407, NCT04409834

Rivaroxaban 
Table 1 (cont.) | Clinical registries and trials studying COVID-19 with cardiovascular implications

\begin{tabular}{|c|c|}
\hline Registry, trial or drug name & NCT identifier \\
\hline \multicolumn{2}{|c|}{ Antiplatelet or anticoagulant therapy (cont.) } \\
\hline Enoxaparin & $\begin{array}{l}\text { NCT04406389, NCT04409834, } \\
\text { NCT04508439, NCT04646655, NCT04394377 }\end{array}$ \\
\hline Unfractionated heparin & $\begin{array}{l}\text { NCT04406389, NCT04409834, } \\
\text { NCT04372589, NCT04394377 }\end{array}$ \\
\hline Fondaparinux & NCT04406389 \\
\hline ‘Therapeutic anticoagulation’ & NCT04444700, NCT04486508 \\
\hline \multicolumn{2}{|l|}{ Lipid-lowering therapy } \\
\hline Atorvastatin & $\begin{array}{l}\text { NCT04333407, NCT04466241, } \\
\text { NCT04380402, NCT04900155 }\end{array}$ \\
\hline Rosuvastatin & NCT04359095 \\
\hline \multicolumn{2}{|c|}{ Pulmonary hypertension and heart failure therapy } \\
\hline Ambrisentan & NCT04393246 \\
\hline Sildenafil & NCT04489446 \\
\hline Dapagliflozin & NCT04393246, NCT04350593 \\
\hline
\end{tabular}

Interestingly, when examining arrhythmia frequency on a global level, regional differences have been reported in the frequency of different arrhythmias ${ }^{44}$.

Atrial fibrillation is the most common arrhythmia reported in acute COVID-19 $\left(\right.$ REF. $\left.^{45}\right)$ and is often of new onset ${ }^{46}$. One study found that the rate of atrial fibrillation in patients with acute COVID-19 is similar to that observed in patients with influenza ${ }^{47}$, and the presence of atrial fibrillation portends a worse outcome ${ }^{46}$. Whether patients with newly diagnosed atrial fibrillation during acute COVID-19 will have recurrent episodes of atrial fibrillation and whether the rate of more malignant arrhythmias is increased in survivors of COVID-19 is uncertain. Reports have documented cases of postural orthostatic tachycardia syndrome as a cause of palpitations after recovery from acute COVID-19, as has been described after other viral illnesses ${ }^{48}$.

QT interval prolongation has been noted in patients hospitalized with COVID-19, including those showing progression to torsades de pointes ${ }^{49}$. Some degree of QT interval prolongation can occur in any patient with COVID-19 but this feature was often exacerbated early in the pandemic when medications with known QT-prolonging adverse effects, such as chloroquine, hydroxychloroquine and azithromycin, were used to treat patients ${ }^{50,51}$.

\section{Cardiac injury in mild acute COVID-19}

Owing to concerns about myocardial injury caused by COVID-19, investigators started imaging survivors of COVID-19 using cardiac MRI. Initial studies demonstrated that, in both selected patients ${ }^{52}$ (those with COVID-19 and elevated troponin levels during hospitalization) and unselected patients ${ }^{53}$ (those with a range of asymptomatic, mild, moderate or severe COVID-19), a concerningly high frequency (up to 78\%) of patients had abnormalities seen on cardiac MRI. These abnormalities included elevated $\mathrm{T} 1$ values (a marker of fibrosis or inflammation) in up to $73 \%$ of patients, increased $\mathrm{T} 2$ values (a marker of oedema) in up to $60 \%$ of patients and myocarditis-like late gadolinium enhancement (LGE) patterns in $32-45 \%$ of patients when cardiac MRI was performed within 37-71 days after COVID-19 diagnosis ${ }^{52,53}$. These findings raised the spectre of ongoing myocarditis and an eventual epidemic of long-term HF. However, these cardiac MRI findings did not correlate with biomarker data.

The concerning results from these studies led to additional investigations with cardiac MRI in patients with a range of COVID-19 severity. Results from these additional studies $^{30,54-66}$ are summarized in TABLE 2 and were not always consistent with the initial studies. Although cardiac MRI still identified patients with myocarditis-like imaging patterns at levels higher than in the general population, the rates were less common than the initial studies suggested. A study by Joy et al. offered further reassurance; they examined 74 seropositive health-care workers who survived COVID-19 at a time point of 6 months after diagnosis ${ }^{64}-$ the longest time point in the literature to date. Abnormal imaging findings were uncommon but present in some survivors of COVID-19, including $4 \%$ with LGE myocardial scarring. Neither LGE myocardial scarring nor any other end point (left ventricular ejection fraction, right ventricular ejection fraction, indexed end-diastolic volume, percentage of LGE, septal or global T1, septal or global $\mathrm{T} 2$, left ventricular mass indexed, left atrial area indexed, global longitudinal shortening, septal extracellular volume and aortic distensibility) was significantly different between the survivors of COVID-19 and matched healthy controls ${ }^{64}$. The researchers also found no significant difference in the levels of N-terminal pro-B-type natriuretic peptide or high-sensitivity troponin between the groups.

A specific group of patients who warrant special consideration are competitive athletes because even a mild decrease in cardiac function that might be asymptomatic in a normal individual can have a substantial effect on the ability of athletes to perform. This point also has implications for 'return to play' because even 
asymptomatic cases of myocarditis can lead to sudden death in athletes ${ }^{67}$. Therefore, several of the above-mentioned studies have focused on collegiate and professional athletes $^{5,55,58-62,65,66}$. Rajpal et al. found that $15 \%$ of college athletes had evidence of a myocarditis-like LGE pattern on cardiac $\mathrm{MRI}^{54}$ but subsequent studies often found no evidence in any athlete, and a large study of 1,597 college athletes with COVID-19 found only $2.3 \%$ with myocarditis, most of whom were asymptomatic ${ }^{66}$. When examining healthy athletes as matched controls in addition to non-athlete healthy controls, these subsequent studies found that focal inferoseptal right ventricle insertion LGE is common among healthy athletes probably caused by remodelling from athletic training - and that this finding should not be confused with myocarditis. The implications of these findings in athletes have been summarized and discussed in detail previously ${ }^{67}$.

One additional imaging measure worth noting is first-phase ejection fraction (EF1) measured by echocardiography, which is a sensitive measure of preclinical HF. Gu et al. examined EF1 in patients with COVID-19 in both Wuhan, China, and London, UK, and found that EF1 was a better predictor of survival than other echocardiographic findings, laboratory findings, age or comorbidities $^{68}$.

Taken together, a variety of laboratory and imaging abnormalities are seen in post-acute COVID-19, although many of the cardiac MRI findings are not detected at a significantly higher rate than among healthy controls in some studies. Of note, the only study that focused beyond the 1-3-month period after diagnosis found no significant differences in any cardiac MRI parameters between survivors of COVID-19 and controls. Furthermore, none of these studies found correlations between abnormal cardiac MRI findings and biomarker or tissue abnormalities. Indeed, the latest studies are much more reassuring, suggesting that the earlier findings were probably overinterpreted, and follow-up of asymptomatic patients with COVID-19 and imaging abnormalities has largely shown preserved left ventricular function ${ }^{43,59,61}$. Long-term studies will be needed to monitor functional status and observe these imaging abnormalities. In summary, the spectre of a looming $\mathrm{HF}$ epidemic caused by COVID-19-induced myocarditis seems much less likely than was feared approximately 1 year ago. Multiple registries are actively collecting information on these patients. Laboratory and imaging abnormalities seen in post-acute COVID-19 are summarized in BOX 1.

\section{Cardiac injury in moderate-to-severe COVID-19}

Multiple early studies demonstrated that patients with COVID-19 and acute myocardial injury, defined as an elevated

Acute COVID-19 illness

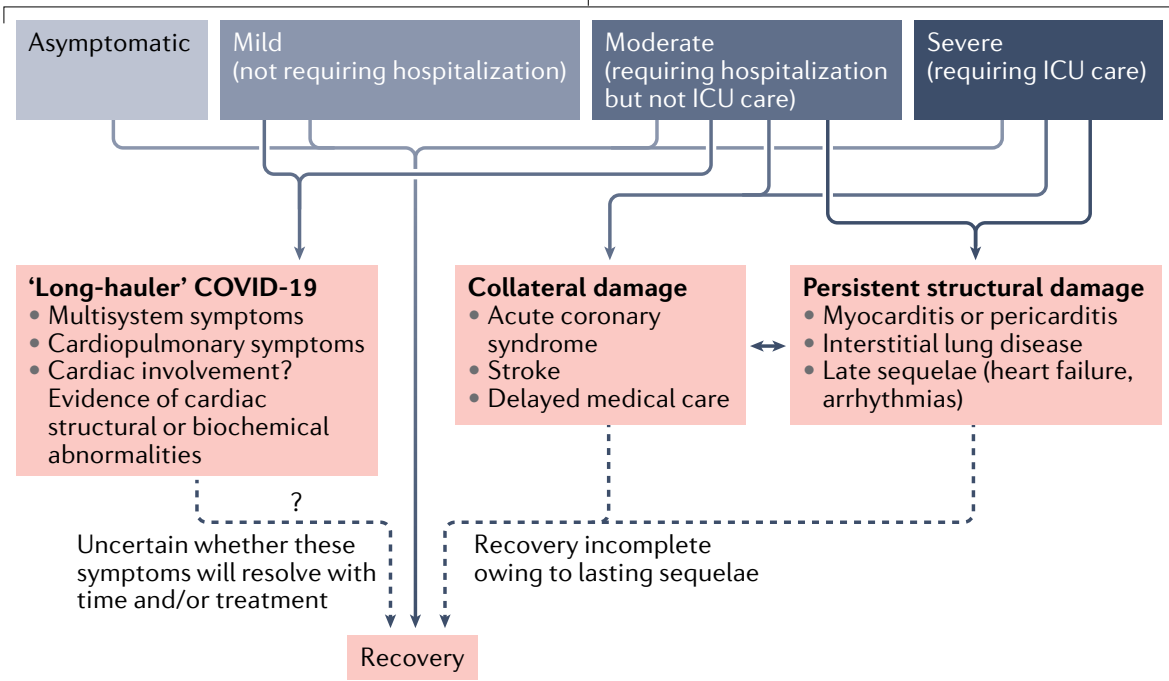

Fig. 2 | The road to recovery in survivors of COVID-19. Some patients recover quickly from acute coronavirus disease 2019 (COVID-19), whereas others have a protracted course due to sequelae from collateral damage of the pandemic, persistent structural damage to the heart or the development of 'long-hauler' COVID-19. Whether measurable physiological or pathological changes are associated with long-hauler COVID-19 and whether these survivors will eventually recover completely with time and/or treatment is unknown. The persistence of symptoms will have a potentially large burden across all aspects of medicine for years to come. ICU, intensive care unit.

serum troponin level, have substantially higher mortality than similar patients with COVID-19 and normal troponin levels $s^{3,8,9,52}$. Indeed, among the standard biomarkers of troponin I, B-type natriuretic peptide, $\mathrm{C}$-reactive protein, ferritin and $\mathrm{D}$-dimer, only the troponin I level was found to be associated with 30-day in-hospital mortality ${ }^{69}$. Elevated levels of troponin and C-reactive protein can occur even in patients with mild COVID-19 who did not require hospitalization ${ }^{53}$. Although some cases of elevated troponin levels were linked to obvious causes, such as acute coronary syndrome or pulmonary embolism ${ }^{8,9}$, in many other cases the cause was unclear.

Many of the cardiac MRI studies described above focused primarily on patients with COVID-19 in whom the initial presentation was mild, but some evidence exists that left and right ventricular dysfunction might persist in patients with COVID-19 who have a more severe initial clinical course, with evidence of acute myocardial injury. Additionally, patients with COVID-19 and elevated C-reactive protein or D-dimer levels or lymphopenia had higher myocardial T2 values than those without these abnormal laboratory values; similarly, patients with severe COVID-19 had higher myocardial T2 values than those with mild or moderate COVID-19 (REF. ${ }^{63}$ ). However, one study found that neither the admission level nor the peak level of troponin could be used to predict the development of myocarditis-like findings on cardiac MRI ${ }^{48}$.

\section{Cardiac pathology findings. Although} cardiac MRI abnormalities have been observed in patients with COVID-19, rarely was an endomyocardial biopsy sample obtained, which is the gold standard for the diagnosis of myocarditis. Another approach to estimating the frequency of myocarditis cases is to look at autopsy findings. One study examined 22 published studies and identified 277 autopsied hearts from patients who died from COVID-19 $\left(\mathrm{REF}^{70}{ }^{70}\right.$. This study reported that $7.2 \%$ of the patients who died from COVID-19 had histological evidence of myocarditis; however, most of these samples showed only small foci of inflammatory infiltrates of questionable clinical relevance in the myocardium. Multifocal or diffuse infiltrates with cardiomyocyte injury that would be expected to cause substantial cardiac dysfunction were found in only $1.4 \%$ of the autopsied hearts. Although the cohort of patients who died from COVID-19 was older and had multiple comorbidities - being 
Table 2 | Published cardiac MRI findings in post-acute COVID-19

\begin{tabular}{|c|c|c|c|c|c|c|c|c|}
\hline \multirow[t]{2}{*}{ Study } & \multirow{2}{*}{$\begin{array}{l}\text { Number of } \\
\text { patients }\end{array}$} & \multirow{2}{*}{$\begin{array}{l}\text { Mean } \\
\text { age } \\
\text { (years) }\end{array}$} & \multirow{2}{*}{$\begin{array}{l}\text { Mean time } \\
\text { from COVID-19 } \\
\text { diagnosis to } \\
\text { MRI (days) }\end{array}$} & \multirow{2}{*}{$\begin{array}{l}\text { Severity of } \\
\text { COVID-19 }\end{array}$} & \multicolumn{4}{|c|}{ Cardiac MRI findings } \\
\hline & & & & & $\begin{array}{l}\text { Total } \\
\text { abnormal (\%) }\end{array}$ & $\begin{array}{l}\text { Myocarditis-like } \\
\text { LGE pattern (\%) }\end{array}$ & $\begin{array}{l}\text { Increased T1 } \\
\text { values (\%) }\end{array}$ & $\begin{array}{l}\text { Increased } \\
\text { T2 values (\%) }\end{array}$ \\
\hline $\begin{array}{l}\text { Puntmann } \\
\text { et al. }^{.3}\end{array}$ & 100 & 49 & 71 & $\begin{array}{l}\text { Asymptomatic (18\%), } \\
\text { mild-moderate } \\
(49 \%) \text {, severe (33\%) }\end{array}$ & 78 & 32 & 73 & 60 \\
\hline $\begin{array}{l}\text { Rajpal } \\
\text { et al. }{ }^{54}\end{array}$ & 26 & 20 & 24 & $\begin{array}{l}\text { Asymptomatic (54\%), } \\
\text { mild ( } 46 \%)\end{array}$ & 46 & 15 & NR & 15 \\
\hline Vago et al..$^{55}$ & 12 & 23 & 17 & $\begin{array}{l}\text { Asymptomatic (17\%), } \\
\text { mild ( } 83 \%)\end{array}$ & NR & 0 & NR & NR \\
\hline $\begin{array}{l}\text { Matek } \\
\text { et al. }{ }^{58}\end{array}$ & 26 & 24 & 32 & $\begin{array}{l}\text { Asymptomatic ( } 23 \%), \\
\text { mild ( } 54 \%), \\
\text { moderate (19\%) }\end{array}$ & 19 & 0 & 0 & 4 \\
\hline $\begin{array}{l}\text { Starekova } \\
\text { et al. }{ }^{59}\end{array}$ & 145 & 20 & 15 & $\begin{array}{l}\text { Asymptomatic ( } 17 \%) \text {, } \\
\text { mild (49\%), moderate } \\
(28 \%) \text {, unknown (6\%) }\end{array}$ & $\geq 29$ & 1.4 & NR & NR \\
\hline Clark et al..$^{60}$ & 59 & 20 & 22 & $\begin{array}{l}\text { Asymptomatic (22\%), } \\
\text { mild (78\%) }\end{array}$ & $\geq 39$ & 3 & NR & NR \\
\hline $\begin{array}{l}\text { Martinez } \\
\text { et al. } .1\end{array}$ & 27 & 25 & 19 & $\begin{array}{l}\text { Asymptomatic (42\%), } \\
\text { mild (58\%) }\end{array}$ & $\geq 19$ & 11 & NR & NR \\
\hline $\begin{array}{l}\text { Moulson } \\
\text { et al. }{ }^{62}\end{array}$ & 317 & 20 & 33 & $\begin{array}{l}\text { Asymptomatic (33\%), } \\
\text { mild ( } 29 \%), \\
\text { moderate (38\%) }\end{array}$ & $\geq 7$ & 5 & NR & NR \\
\hline $\begin{array}{l}\text { Hendrickson } \\
\text { et al. }{ }^{65}\end{array}$ & 5 & 20 & 16 & $\begin{array}{l}\text { Asymptomatic (18\%), } \\
\text { mild ( } 55 \%) \text {, } \\
\text { moderate }(27 \%)\end{array}$ & 0 & 0 & 0 & 0 \\
\hline $\begin{array}{l}\text { Daniels } \\
\text { et al. }{ }^{66}\end{array}$ & 1,597 & NR & 28 & NR & $\geq 2.3$ & 2.3 & $\geq 0.3$ & $\geq 1.9$ \\
\hline
\end{tabular}

COVID-19, coronavirus disease 2019; LGE, late gadolinium enhancement; NR, not reported.

distinct from the population of young, relatively healthy survivors in which some concerning reports of myocarditis have been published - a myocarditis rate of 1.4-7.2\% is far below that reported in the initial imaging studies and is in accordance with the $2.3 \%$ rate of myocarditis reported in a large imaging study by Daniels et al. ${ }^{66}$.

\section{Recovery from moderate-to-severe acute} COVID-19. The available evidence suggests that patients with COVID-19 requiring hospitalization, regardless of age, have worse long-term outcomes than those who did not require hospitalization ${ }^{71,72}$. However, data on these outcomes are still scarce and more information is urgently needed. The percentage of patients who have persistently elevated troponin levels, the timeline for eventual normalization of troponin levels, and the clinical relevance of persistently elevated troponin levels with left ventricular dysfunction or other prognostic indicators all remain uncertain. Acutely decreased left ventricular systolic function associated with moderate-to-severe COVID-19 occurs from a variety of causes, including myocarditis, stress-induced cardiomyopathy, critical illness cardiomyopathy and acute myocardial infarction. Anecdotal experience as well as a small number of published studies ${ }^{73-76}$ suggest that, in many of these cases, the ventricular dysfunction is only temporary and returns to normal within days to weeks. However, this recovery has not been well documented in the literature and the long-term prognosis for either those with recovery of left ventricular function or those without is not known.

Patients with COVID-19 and evidence of acute cardiac injury should be monitored for long-term prognosis. Compared with those who never required hospitalization, more baseline laboratory and imaging information is available in survivors of moderate-to-severe COVID-19, but there is a dearth of long-term follow-up data specifically on the development of HF and arrhythmias. These patients should be 
enrolled in longitudinal follow-up studies to understand how abnormal laboratory and imaging findings progress.

Probable long-term sequelae. Owing to the high rate of clinically significant cardiovascular events that can occur during acute COVID-19, including acute myocardial infarction ${ }^{2}$, stroke ${ }^{77}$, venous thromboembolism $^{78}$ and the development of arrhythmias $^{44,79}$, certain long-term sequelae are expected. Increased rates of systolic dysfunction and clinical HF are likely to occur from delayed presentation to hospital with acute myocardial infarction and possibly related to myocarditis and stress cardiomyopathy. In addition, we are seeing typical neurological sequelae from COVID-19-related stroke. Survivors of COVID-19 have the potential to develop pulmonary hypertension secondary to venous thromboembolic disease ${ }^{80,81}$. The need for interventions to control cardiac arrhythmias, including ablation procedures and/or placement of implantable cardioverter-defibrillators, might increase. Additionally, an overlap might exist between postural orthostatic tachycardia syndrome, chronic fatigue syndrome and post-acute COVID-19 syndrome s8,82 $^{48}$.

\section{Delays in cardiovascular care}

The COVID-19 pandemic greatly disrupted how patients and the health-care system interacted, especially during the first months of the pandemic. Many patients delayed urgent and emergent care, including for acute cardiovascular conditions. Hospitalizations temporarily decreased ${ }^{83}$, but, in the case of acute coronary syndromes, the pandemic often simply led to a delayed presentation to hospital ${ }^{84}$, which is associated with worse outcomes ${ }^{85}$. Indeed, a multicentre, international registry of patients with COVID-19 and suspected

\section{Box 1 | Laboratory and imaging abnormalities in COVID-19}

Various laboratory and imaging abnormalities are observed in both acute and post-acute coronavirus disease 2019 (COVID-19). Long-term studies are needed to understand the implications of these findings and whether they resolve or result in lasting sequelae.

\section{Laboratory abnormalities}

- Cardiac biomarkers: elevated troponin and $\mathrm{N}$-terminal pro-B-type natriuretic peptide levels

- Cell counts: lymphopenia, leukocytosis, neutrophilia and thrombocytopenia

- Inflammatory markers: elevated erythrocyte sedimentation rate and C-reactive protein, ferritin, IL-6 and lactate dehydrogenase levels

- Coagulation markers: elevated D-dimer and fibrinogen levels and prolonged prothrombin time and activated partial thromboplastin clotting time

\section{Echocardiographic abnormalities}

- Decreased systolic function (left and/or right ejection fraction)

- Ventricular wall hypokinesis or dyskinesis

- Increased left ventricular volume

- Pericardial effusion (see the figure, panel a, arrow)

\section{MRI abnormalities}

- Late gadolinium enhancement of epicardium, myocardium or endocardium (see the figure, panel b, arrows)

- Late gadolinium enhancement of the pericardium

- Abnormalities in T1-weighted and/or T2-weighted images
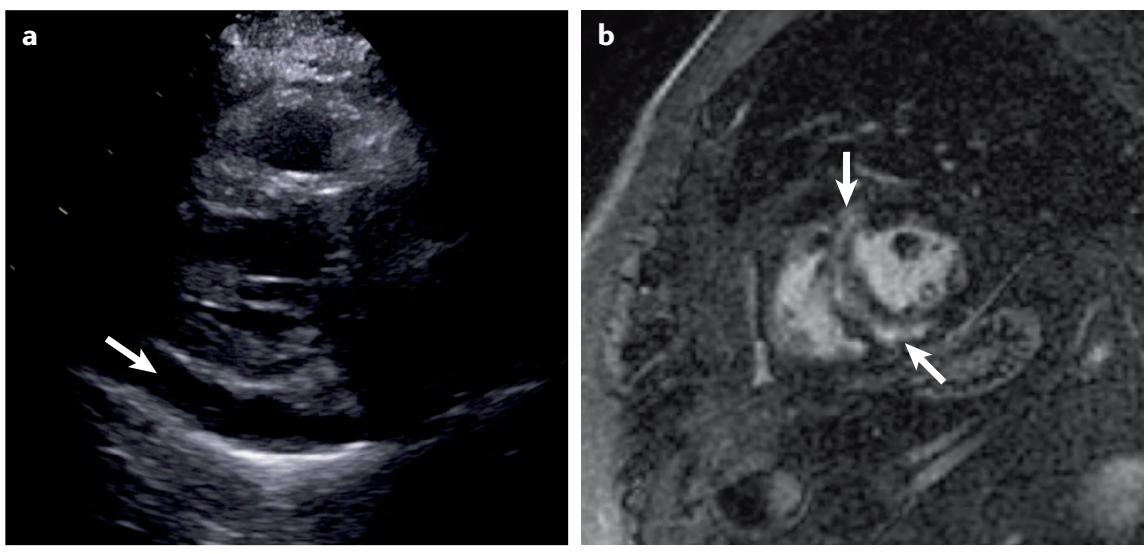

acute coronary syndrome who underwent invasive coronary angiography found that symptom-to-admission times were greatly increased and that in-hospital mortality was higher among patients with any type of acute coronary syndrome than in recent pre-pandemic times; cardiogenic shock was also much more common ${ }^{86}$. The North American COVID-19 and STEMI (NACMI) registry similarly found that patients with ST-segment elevation myocardial infarction and COVID-19 were at higher risk of a composite end point of in-hospital death, stroke, recurrent myocardial infarction or repeat revascularization than they were before the pandemic ${ }^{87}$. These patients also presented with higher rates of cardiogenic shock and were less likely to receive invasive angiography than control patients from the pre-COVID era ${ }^{87}$. Although not yet documented, these survivors of an acute coronary syndrome are very likely to have a higher rate of long-term complications, including $\mathrm{HF}$ and arrhythmias, even in those who did present to hospital, owing to increased door-to-balloon times ${ }^{88,89}$. Furthermore, postponed outpatient preventive care appointments might have contributed to worse long-term outcomes, although this effect will be difficult to quantify ${ }^{90}$.

\section{Complications exacerbated in racial/ethnic} minorities. Throughout much of the developed world, health disparities related to race/ethnicity have been exacerbated by the COVID-19 pandemic. In the USA ${ }^{91-93}, \mathrm{UK}^{94}$ and other developed countries, racial/ethnic minorities have significantly higher rates of SARS-CoV-2 infection and COVID-19 hospitalization and mortality. These groups are also known to have higher baseline levels of cardiovascular comorbidities ${ }^{92}$, obesity ${ }^{95}$, diabetes mellitus ${ }^{96}$ and chronic kidney disease $^{97}$, which puts them at even higher risk of more severe acute illness and/or adverse COVID-19 outcomes. Importantly, these groups also tend to have lower access to health care. Of note, the NACMI registry found that ST-segment elevation myocardial infarction among patients with COVID-19 disproportionately affected racial/ethnic minorities in the USA ${ }^{87}$. Published data on racial/ethnic differences in cardiovascular outcomes during the COVID-19 pandemic are limited, but this topic warrants further investigation.

\section{Cardiac complications after COVID-19 vaccination}

Rare cases of pericarditis or myocarditis have been reported after vaccination with various vaccines, best studied in live-virus 
vaccines, such as those for measles/ mumps/rubella, varicella zoster virus, yellow fever or smallpox ${ }^{98}$. Similarly, rare cases of pericarditis or myocarditis have been reported after the second dose of the COVID-19 vaccine, typically in young men $^{99,100}$. Although vaccine-associated pericarditis or myocarditis has received widespread attention in the media, this adverse effect is quite rare, with an initial report showing approximately 12.6 cases per 1,000,000 (second) doses among young adults (aged 12-39 years) ${ }^{101}$. More recent data suggest that the incidence might be tenfold higher, but this complication is still rare, and most cases are mild ${ }^{102,103}$. The severity of the complication tends to be mild to moderate and is diagnosed clinically and with cardiac MRI, although rare reports of fulminant myocarditis with histopathology have been reported ${ }^{104}$. The long-term implications in these patients are not yet known, but this small risk is thought to be far outweighed by the benefit of vaccination against COVID-19. One study estimated that COVID-19 vaccination of the group of individuals who were most likely to experience post-vaccination myocarditis (adolescent and young adult men) would prevent $>10$ times the number of hospitalizations and 3-4 times the number of ICU admissions owing to COVID-19 than would result from cases of vaccine-associated myocarditis ${ }^{105}$.

\section{Future investigations}

Although no data are so far available on long-term cardiovascular sequelae, given the disease manifestations and pathogenesis observed during acute COVID-19, we can make educated determinations of certain cardiovascular complications that should undergo prospective monitoring in survivors of COVID-19 (BOX 2). Specific areas to investigate should include correlations between imaging abnormalities and pathology or histology, clinical syndromes and the development of long-term comorbidities. These studies should aim to determine the mechanisms and aetiology of different manifestations of viral presence in the heart. We might reasonably predict increased rates of progression to atherosclerotic cardiovascular disease, $\mathrm{HF}$ and/or valvular disease owing to lasting inflammatory effects, and these outcomes should be specifically assessed. Although cardiopulmonary symptoms are common in post-acute COVID-19, determining whether these symptoms are linked to objective evidence of cardiovascular disease by electrocardiography, echocardiography

\section{Box 2 | Cardiac monitoring in survivors of COVID-19}

- In patients with coronavirus disease 2019 (COVID-19) and elevated troponin levels, how long does troponin take to normalize?

- In patients with COVID-19 and newly reduced left ventricular ejection fraction (LVEF), how many normalize and how long does this normalization take?

- Is there an association between normalization of troponin levels and normalization of LVEF if both were abnormal during acute COVID-19?

- In patients with acute COVID-19 who present with reduced LVEF or abnormal first-phase ejection fraction but in whom these parameters subsequently normalize, is there an increased risk of developing heart failure in the coming years?

- In patients with a myocarditis-like late gadolinium enhancement pattern or abnormal T1 or T2 values on cardiac MRI, is there an increased risk of developing heart failure in the coming years? - Are survivors of COVID-19 at increased risk of diastolic dysfunction over the long term?

or advanced imaging will be important. Determining whether the presence of these symptoms is associated with worse prognosis will also be key.

End points of these clinical studies should include functional status and symptoms, survival and cardiac morbidity (that is, the development of HF or arrhythmias). These end points should be assessed by imaging with cardiac MRI, echocardiography and/or PET as well as by the use of stress testing, biomarker measuring and event monitoring.

Given that few data are available on how these cardiovascular complications should be managed, we recommend that clinical judgement be used to determine follow-up and management in a manner similar to if they had occurred due to other, better-studied aetiologies. One useful guide for clinicians could be a position paper from the $\mathrm{ESC}^{106}$.

\section{Conclusions}

More than 18 months have passed since the WHO announced the pandemic nature of COVID-19. In that time, we have learned that cardiac injury is common during acute COVID-19 and that it is associated with worse short-term outcomes, that the aetiology of cardiac injury is multifactorial and that imaging abnormalities are common irrespective of the severity of the acute illness. However, much remains to be learned about the natural history and clinical importance of these findings in survivors of acute COVID-19, especially those with post-acute COVID-19 syndrome. We need more information on correlations between imaging findings and pathology and histology. We do not understand the aetiology of different manifestations of SARS-CoV-2 presence in cardiac tissues. The window of opportunity is quite narrow as cases of acute COVID-19 infection decrease with the roll-out of vaccination programmes. Governments, academic centres and private-public collaborations must invest the time and resources into this endeavour to understand the complex and disparate natural history of cardiovascular disease in the large number of survivors of COVID-19 as well as to prepare for the next potential pandemic.

\section{Benjamin A. Satterfield (iD) ${ }^{1}$, Deepak L. Bhatt (iD) 2 and Bernard J. Gersh iD ${ }^{1 凶}$}

'Department of Cardiovascular Medicine, Mayo Clinic, Rochester, MN, USA.

${ }^{2}$ Department of Cardiovascular Medicine, Brigham and Women's Hospital, Harvard Medical School, Boston, MA, USA.

凶e-mail: gersh.bernard@mayo.edu

https://doi.org/10.1038/s41569-021-00631-3

Published online 22 October 2021

1. Dehingia, N. \& Raj, A. Sex differences in COVID-19 case fatality: do we know enough? Lancet Glob. Health 9, e14-e15 (2021)

2. Bavishi, C. et al. Acute myocardial injury in patients hospitalized with COVID-19 infection: a review. Prog. Cardiovasc. Dis. 63, 682-689 (2020).

3. Guo, T. et al. Cardiovascular implications of fatal outcomes of patients with coronavirus disease 2019 (COVID-19). JAMA Cardiol. 5, 811-818 (2020).

4. Long, B., Brady, W. J., Koyfman, A. \& Gottlieb, M. Cardiovascular complications in COVID-19. Am. J. Emerg. Med. 38, 1504-1507 (2020)

5. Manolis, A. S., Manolis, A. A., Manolis, T. A. \& Melita, H. COVID-19 and acute myocardial injury and infarction: related mechanisms and emerging challenges. J. Cardiovasc. Pharmacol. Ther. 26, 399-414 (2021).

6. Friedrich, M. G. \& Cooper, L. T. Jr What we (don't) know about myocardial injury after COVID-19. Eur. Heart J. 42, 1879-1882 (2021).

7. Bularga, A., Chapman, A. R. \& Mills, N. L. Mechanisms of myocardial injury in COVID-19. Clin. Chem. 67, 1044-1046 (2021).

8. Shi, S. et al. Characteristics and clinical significance of myocardial injury in patients with severe coronavirus disease 2019. Eur. Heart J. 41, 2070-2079 (2020).

9. Wei, J.-F. et al. Acute myocardial injury is common in patients with COVID-19 and impairs their prognosis. Heart 106, 1154-1159 (2020).

10. Siddiqi, H. K. et al. Increased prevalence of myocardial injury in patients with SARS-CoV-2 viremia. Am. J. Med. 134, 542-546 (2021).

11. Nalbandian, A. et al. Post-acute COVID-19 syndrome. Nat. Med. 27, 601-615 (2021).

12. Satterfield, B. A., Dikilitas, O. \& Kullo, I. J. Leveraging the electronic health record to address the COVID-19 pandemic. Mayo Clin. Proc 96, 1592-1608 (2021).

13. Vaduganathan, M. et al. Regulation of cardiovascular therapies during the COVID-19 public health emergency. J. Am. Coll. Cardiol. 76, 2517-2521 (2020). 
14. Gaba, P. \& Bhatt, D. L. The COVID-19 pandemic: a catalyst to improve clinical trials. Nat. Rev. Cardiol. 17, 673-675 (2020)

15. Wang, X. \& Bhatt, D. L. COVID-19: an unintended force for medical revolution. J. Invasive Cardiol. 32 E81-E82 (2020).

16. Selvaraj, S. et al. The landscape of cardiovascular clinical trials in the United States initiated before and during COVID-19. J. Am. Heart Assoc. 9, e018274 (2020).

17. López-Medina, E. et al. Effect of ivermectin on time to resolution of symptoms among adults with mild COVID-19: a randomized clinical trial. JAMA 325 1426-1435 (2021)

18. Sadeghipour, P. et al. Effect of intermediate-dose vs standard-dose prophylactic anticoagulation on thrombotic events, extracorporeal membrane oxygenation treatment, or mortality among patients with COVID-19 admitted to the intensive care unit: the INSPIRATION randomized clinical trial. JAMA 325, 1620-1630 (2021).

19. Zhang, G., Wu, Y., Xu, R. \& Du, X. Effects of reninangiotensin-aldosterone system inhibitors on disease severity and mortality in patients with COVID-19: a meta-analysis. J. Med. Virol. 93, 2287-2300 (2021).

20. Bagiella, E., Bhatt, D. L. \& Gaudino, M. The consequences of the COVID-19 pandemic on non-COVID-19 clinical trials. J. Am. Coll. Cardiol. 76 342-345 (2020)

21. Ahmed, $\mathbf{H}$. et al. Long-term clinical outcomes in survivors of severe acute respiratory syndrome (SARS) and Middle East respiratory syndrome (MERS) coronavirus outbreaks after hospitalisation or ICU admission: a systematic review and meta-analysis J. Rehabil. Med. 52, jrm00063 (2020).

22. Hui, D. et al. Impact of severe acute respiratory syndrome (SARS) on pulmonary function, functional capacity and quality of life in a cohort of survivors. Thorax 60, 401-409 (2005)

23. Lam, M. H.-B. et al. Mental morbidities and chronic fatigue in severe acute respiratory syndrome survivors: long-term follow-up. Arch. Intern. Med. 169, 2142-2147 (2009).

24. Lee, S. H. et al. Depression as a mediator of chronic fatigue and post-traumatic stress symptoms in middle east respiratory syndrome survivors. Psychiatry Investig. 16, 59-64 (2019).

25. Moldofsky, H. \& Patcai, J. Chronic widespread musculoskeletal pain, fatigue, depression and disordered sleep in chronic post-SARS syndrome; a case-controlled study. BMC Neurol. 11, 37 (2011).

26. Ong, K. C. et al. Pulmonary function and exercise capacity in survivors of severe acute respiratory syndrome. Eur. Respir. J. 24, 436-442 (2004).

27. Lee, A. M. et al. Stress and psychological distress among SARS survivors 1 year after the outbreak. Can. J. Psychiatry 52, 233-240 (2007).

28. Datta, S. D., Talwar, A. \& Lee, J. T. A proposed framework and timeline of the spectrum of disease due to SARS-CoV-2 infection: Illness beyond acute infection and public health implications. JAMA 324 2251-2252 (2020)

29. Greenhalgh, T., Knight, M., Buxton, M. \& Husain, L. Management of post-acute Covid-19 in primary care. BMJ 370, m3026 (2020)

30. Huang, L. et al. Cardiac involvement in patients recovered from COVID-2019 identified using magnetic resonance imaging. JACC Cardiovasc. Imaging 13 , 2330-2339 (2020)

31. Gorna, R. et al. Long COVID guidelines need to reflect lived experience. Lancet 397, 455-457 (2021).

32. Logue, J. K. et al. Sequelae in adults at 6 months after COVID-19 infection. JAMA Netw. Open. 4, e210830 (2021).

33. Carfi, A., Bernabei, R. \& Landi, F. Persistent symptoms in patients after acute COVID-19. JAMA 324, 603-605 (2020).

34. Carvalho-Schneider, C. et al. Follow-up of adults with noncritical COVID-19 two months after symptom onset. Clin. Microbiol. Infect. 27, 258-263 (2021).

35. Huang, C. et al. 6-Month consequences of COVID-19 in patients discharged from hospital: a cohort study. Lancet 397, 220-232 (2021).

36. Romero-Duarte, Á. et al. Sequelae, persistent symptomatology and outcomes after COVID-19 hospitalization: the ANCOHVID multicentre 6-month follow-up study. BMC Med. 19, 129 (2021).

37. Maltezou, H. C., Pavli, A. \& Tsakris, A. Post-COvID syndrome: an insight on its pathogenesis. Vaccines $\mathbf{9}$, 497 (2021).
38. Babapoor-Farrokhran, S., Rasekhi, R. T., Gill, D., Babapoor, S. \& Amanullah, A. Arrhythmia in COVID-19. SN Compr. Clin. Med. 2, 1430-1435 (2020).

39. Kochav, S. M. et al. Cardiac arrhythmias in COVID-19. Infect. Circ. Arrhythm. Electrophysiol. 13, e008719 (2020).

40. Wang, D. et al. Clinical characteristics of 138 hospitalized patients with 2019 novel coronavirusinfected pneumonia in Wuhan, China. JAMA 323, 1061-1069 (2020).

41. Goyal, P. et al. Clinical characteristics of Covid-19 in New York City. N. Engl. J. Med. 382, 2372-2374 (2020).

42. Colon, C. M. et al. Atrial arrhythmias in COVID-19 patients. Clin. Electrophysiol. 6, 1189-1190 (2020).

43. Zhou, M. et al. Cardiovascular sequalae in uncomplicated COVID-19 survivors. PLoS One 16 e0246732 (2021).

44. Coromilas, E. J. et al. Worldwide survey of COVID-19associated arrhythmias. Circ. Arrhythm. Electrophysiol. 14, e009458 (2021).

45. Gopinathannair, R. et al. COVID-19 and cardiac arrhythmias: a global perspective on arrhythmia characteristics and management strategies. J. Interv. Card. Electrophysiol. 59, 329-336 (2020).

46. Spinoni, E. G. et al. Contribution of atrial fibrillation to in-hospital mortality in patients with COVID-19. Circ. Arrhythm. Electrophysiol. 14, e009375 (2021).

47. Musikantow, D. R. et al. Atrial fibrillation in patients hospitalized with COVID-19: incidence, predictors, outcomes and comparison to influenza. JACC Clin Electrophysiol. 7, 1120-1130 (2021).

48. Blitshteyn, S. \& Whitelaw, S. Postural orthostatic tachycardia syndrome (POTS) and other autonomic disorders after COVID-19 infection: a case series of 20 patients. Immunol. Res. 69, 205-211 (2021).

49. Rubin, G. A et al. Cardiac corrected OT interval changes among patients treated for COVID-19 infection during the early phase of the pandemic. JAMA Netw. Open 4, e216842 (2021).

50. O'Connell, T. F. et al. Hydroxychloroquine/azithromycin therapy and OT prolongation in hospitalized patients with COVID-19. Clin. Electrophysiol. 7, 16-25 (2021)

51. Oren, O. et al. Use of chloroquine and hydroxychloroquine in COVID-19 and cardiovascular implications: understanding safety discrepancies to improve interpretation and design of clinical trials. Circ. Arrhythm. Electrophysiol. 13, e008688 (2020).

52. Knight, D. S. et al. COVID-19: myocardial injury in survivors. Circulation 142, 1120-1122 (2020).

53. Puntmann, V. O. et al. Outcomes of cardiovascular magnetic resonance imaging in patients recently recovered from coronavirus disease 2019 (COVID-19). JAMA Cardiol. 5, 1265-1273 (2020)

54. Rajpal, S. et al. Cardiovascular magnetic resonance findings in competitive athletes recovering from COVID-19 infection. JAMA Cardiol. 6, 116-118 (2021).

55. Vago, H., Szabo, L., Dohy, Z. \& Merkely, B. Cardiac magnetic resonance findings in patients recovered from COVID-19: initial experiences in elite athletes. IJACC Cardiovasc. Imaging 14, 1279-1281 (2020)

56. Kotecha, T. et al. Patterns of myocardial injury in recovered troponin-positive COVID-19 patients assessed by cardiovascular magnetic resonance. Eur. Heart J. 42, 1866-1878 (2021)

57. Raman, B. et al. Medium-term effects of SARS-CoV-2 infection on multiple vital organs, exercise capacity, cognition, quality of life and mental health, posthospital discharge. EClinicalMedicine 31, 100683 (2021).

58. Małek, A et al Cardiac involvement in consecutive elite athletes recovered from Covid-19: a magnetic resonance study. J. Magn. Reson. Imaging 53, 1723-1729 (2021).

59. Starekova, J. et al. Evaluation for myocarditis in competitive student athletes recovering from coronavirus disease 2019 with cardiac magnetic resonance imaging. JAMA Cardiol. 6, 945-950 (2021).

60. Clark, D. E. et al. COVID-19 myocardial pathology evaluation in AthleTEs with cardiac magnetic resonance (COMPETE CMR). Circulation 143 609-612 (2021).

61. Martinez, M. W. et al. Prevalence of inflammatory heart disease among professional athletes with prio COVID-19 infection who received systematic returnto-play cardiac screening. JAMA Cardiol. 6, 745-752 (2021).

62. Moulson, N. et al. SARS-CoV-2 cardiac involvement in young competitive athletes. Circulation 144, 256-266 (2021).
63. Pan, C. et al. Cardiac T1 and T2 mapping showed myocardial involvement in recovered COVID-19 patients initially considered devoid of cardiac damage. J. Magn. Reson. Imaging 54, 421-428 (2021).

64. Joy, G. et al. Prospective case-control study of cardiovascular abnormalities 6 months following mild COVID-19 in healthcare workers. IJACC CardiovasC. Imaging https://doi.org/10.1016/j.jcmg.2021.04.011 (2021).

65. Hendrickson, B. S. et al. Cardiovascular evaluation after COVID-19 in 137 collegiate athletes: results of an algorithm-guided screening. Circulation 143, 1926-1928 (2021).

66. Daniels, C. J. et al. Prevalence of clinical and subclinical myocarditis in competitive athletes with recent SARS-CoV-2 infection: results from the Big Ten COVID-19 Cardiac Registry. JAMA Cardiol. 6, 1078-1087 (2021).

67. Udelson, J. E., Rowin, E. J. \& Maron, B. J. Return to play for athletes after COVID-19 infection: the fog begins to clear. JAMA Cardiol. 6, 997-999 (2021).

68. Gu, H. et al. First-phase ejection fraction, a measure of preclinical heart failure, is strongly associated with increased mortality in patients with COVID-19. Hypertension 77, 2014-2022 (2021).

69. Manocha, K. K. et al. Troponin and other biomarker levels and outcomes among patients hospitalized with COVID-19: derivation and validation of the HA2T2 COVID-19 mortality risk score. J. Am. Heart Assoc. 10, e018477 (2021).

70. Halushka, M. K. \& Vander Heide, R. S. Myocarditis is rare in COVID-19 autopsies: cardiovascular findings across 277 post-mortem examinations. Cardiovasc. Pathol. 50, 107300 (2021).

71. Gallo Marin, B. et al. Predictors of COVID-19 severity: a literature review. Rev. Med. Virol. 31, 1-10 (2021).

72. Cunningham, J. W. et al. Clinical outcomes in young US adults hospitalized with COVID-19. JAMA Intern Med. 181, 379-381 (2021)

73. Jacquet-Lagrèze, M., Riad, Z., Hugon-Vallet, E., Ferraris, A. \& Fellahi, J.-L. Left ventricular dysfunction in COVID-19: a diagnostic issue. Anaesth. Crit. Care Pain. Med. 39, 393 (2020).

74. Tsao, C. W., Strom, J. B., Chang, J. D. \& Manning, W. J. COVID-19-associated stress (Takotsubo) cardiomyopathy. Circ. Cardiovasc Imaging 13, e011222 (2020).

75. Kir, D., Beer, N. \& De Marchena, E. J. Takotsubo cardiomyopathy caused by emotional stressors in the coronavirus disease 2019 (COVID-19) pandemic era. J. Card. Surg. 36, 764-769 (2021).

76. Finsterer, J. \& Stöllberger, C. SARS-CoV-2 triggered Takotsubo in 38 patients. J. Med. Virol. 93 , 1236-1238 (2021).

77. Annie, F., Bates, M. C., Nanjundappa, A., Bhatt, D. L. \& Alkhouli, M. Prevalence and outcomes of acute ischemic stroke among patients $\leq 50$ years of age with laboratory confirmed COVID-19 infection. Am. J. Cardiol. 130, 169-170 (2020)

78. Cohen, S. L. et al. Prevalence and predictors of venous thromboembolism or mortality in hospitalized COVID-19 patients. Thromb. Haemost. 121 1043-1053 (2021).

79. O'Shea, C. J. et al. Ventricular arrhythmia burden during the coronavirus disease 2019 (COVID-19) pandemic. Eur. Heart J. 42, 520-528 (2021).

80. Scudiero, F. et al. Pulmonary embolism in COVID-19 patients: prevalence, predictors and clinical outcome. Thromb. Res. 198, 34-39 (2021).

81. Vlachou, M. et al. Pulmonary thrombosis in Covid-19. before, during and after hospital admission. J. Thromb. Thrombolysis 51, 978-984 (2021).

82. Raj, S. R. et al. Long-COVID postural tachycardia syndrome: an American Autonomic Society statement Clin. Auton. Res. 31, 365-368 (2021).

83. Bhatt, A. S. et al. Fewer hospitalizations for acute cardiovascular conditions during the COVID-19 pandemic. J. Am. Coll. Cardiol. 76, 280-288 (2020).

84. Toner, L. et al. Acute coronary syndromes undergoing percutaneous coronary intervention in the COVID-19 era: comparable case volumes but delayed symptom onset to hospital presentation. Eur. Heart J. Oual. Care Clin. Outcomes 6, 225-226 (2020).

85. Nicholls, M. COVID-19 and cardiovascular disease. Eur. Heart J. 41, 2727-2729 (2020)

86. Kite, T. et al. TCT CONNECT-215 demographics and in-hospital outcomes of COVID-19 patients undergoing an invasive strategy for acute coronary syndrome: the global multi-centre prospective COVID-ACS registry [abstract]. J. Am. Coll. Cardiol. 76, B91 (2020). 
87. Garcia, S. et al. Initial findings from the North American COVID-19 myocardial infarction registry. J. Am. Coll. Cardiol. 77, 1994-2003 (2021).

88. Chew, N. W. et al. Impact of the COVID-19 Pandemic on door-to-balloon time for primary percutaneous coronary intervention - results from the Singapore Western STEMI network. Circ. J. 85, 139-149 (2021).

89. Reinstadler, S. J. et al. Effect of the COVID-19 pandemic on treatment delays in patients with ST-segment elevation myocardial infarction. J. Clin. Med 9, 2183 (2020).

90. Findling, M. G., Blendon, R. J. \& Benson, J. M Delayed care with harmful health consequences reported experiences from national surveys during coronavirus disease 2019. JAMA Health Forum 1 e201463 (2020).

91. Lopez, L., Hart, L. H. \& Katz, M. H. Racial and ethnic health disparities related to COVID-19. JAMA 325 , 719-720 (2021)

92. Aleligne, Y. K., Appiah, D. \& Ebong, I. A. Racial disparities in coronavirus disease 2019 (COVID-19) outcomes. Curr. Opin. Cardiol. 36, 360-366 (2021).

93. Tai, D. B. G., Shah, A., Doubeni, C. A., Sia, I. G $\&$ Wieland, M. L. The disproportionate impact of COVID-19 on racial and ethnic minorities in the United States. Clin. Infect. Dis. 72, 703-706 (2021).

94. Phiri, P., Delanerolle, G., Al-Sudani, A. \& Rathod, S. COVID-19 and black, Asian, and minority ethnic communities: a complex relationship without just cause. JMIR Public Health Surveill. 7, e2258 (2021).

95. Fryar, C. D., Carroll, M. D. \& Ogden, C. Prevalence of Overweight, Obesity, and Severe Obesity Among Adults Age 20 and Over: United States, 1960-1962 Through 2015-2016 (US National Center for Health Statistics, 2020)

96. CDC. National Diabetes Statistics Report, 2020. Estimates of diabetes and its burden in the United States. CDC.gov https://www.cdc.gov/diabetes/pdfs/ data/statistics/national-diabetes-statistics-report.pdf (2020).

97. CDC. Chronic Kidney Disease in the United States. CDC.gov https://www.cdc.gov/kidneydisease/pdf/ Chronic-Kidney-Disease-in-the-US-2021-h.pdf (2021).

98. Kuntz, J., Crane, B., Weinmann, S. \& Naleway, A. L. Myocarditis and pericarditis are rare following live viral vaccinations in adults. Vaccine 36, 1524-1527 (2018).
99. Kim, H. W. et al. Patients with acute myocarditis following mRNA COVID-19 vaccination. JAMA Cardiol. 6, 1196-1201 (2021).

100. Montgomery, J. et al. Myocarditis following immunization with mRNA COVID-19 vaccines in members of the US military. JAMA Cardiol. 6 1202-1206 (2021).

101. Bozkurt, B., Kamat, I. \& Hotez, P. J. Myocarditis with COVID-19 mRNA Vaccines. Circulation 144, 471-484 (2021).

102. Witberg, G. et al. Myocarditis after Covid-19 vaccination in a large health care organization. N. Engl. J. Med. https://doi.org/10.1056/NEJMoa2110737 (2021).

103. Mevorach, D. et al. Myocarditis after BNT162b2 mRNA vaccine against Covid-19 in Israel. N. Engl. J. Med. https://doi.org/10.1056/NEJMoa2109730 (2021).

104. Verma, A. K., Lavine, K. J. \& Lin, C.-Y. Myocarditis after Covid-19 mRNA vaccination. N. Engl. J. Med. 385, 1332-1334 (2021).

105. Dionne A et al Association of myocarditis with BNT162b2 messenger RNA COVID-19 vaccine in a case series of children. JAMA Cardiol. https://doi.org/ 10.1001/jamacardio.2021.3471 (2021).

106. Richter, D. et al. Late phase of COVID-19 pandemic in general cardiology. A position paper of the ESC Council for Cardiology Practice. ESC Heart Fail. 8 , 3483-3494 (2021).

\section{Author contributions}

B.A.S. researched data for the article and wrote the manuscript. D.L.B. and B.J.G. discussed the content of the article and reviewed/edited it before submission.

\section{Competing interests}

D.L.B. declares the following relationships. Advisory Board: Cardax, CellProthera, Cereno Scientific, Elsevier Practice Update Cardiology, Janssen, Level Ex, Medscape Cardiology, MyoKardia, NirvaMed, Novo Nordisk, PhaseBio, PLx Pharma, Regado Biosciences; Board of Directors: Boston VA Research Institute, Society of Cardiovascular Patient Care, TobeSoft: Chair: Inaugural Chair, American Heart Association Quality Oversight Committee; Data Monitoring Committees: Baim Institute for Clinical Research (formerly Harvard Clinical Research Institute, for the PORTICO trial, funded by St. Jude Medical, now Abbott), Cleveland Clinic (including for the ExCEED trial, funded by Edwards), Contego Medical (Chair, PERFORMANCE 2), Duke Clinical Research Institute, Mayo Clinic, Mount Sinai School of Medicine (for the ENVISAGE trial, funded by Daiichi Sankyo), Novartis, Population Health
Research Institute; Honoraria: American College of Cardiology (Senior Associate Editor, Clinical Trials and News, ACC.org; Chair, ACC Accreditation Oversight Committee), Baim Institute for Clinical Research (formerly Harvard Clinical Research Institute; RE-DUAL PCI clinical trial steering committee funded by Boehringer Ingelheim; AEGIS-II executive committee funded by CSL Behring), Belvoir Publications (Editor in Chief, Harvard Heart Letter), Canadian Medical and Surgical Knowledge Translation Research Group (clinical trial steering committees), Duke Clinical Research Institute (clinical trial steering committees, including for the PRONOUNCE trial, funded by Ferring Pharmaceuticals). HMP Global (Editor in Chief, Journal of Invasive Cardiology), Journal of the American College of Cardiology (Guest Editor; Associate Editor), K2P (Co-Chair, interdisciplinary curriculum), Level Ex, Medtelligence/ReachMD (CME steering committees), MJH Life Sciences, Population Health Research Institute (for the COMPASS operations committee, publications committee, steering committee, and USA national co-leader, funded by Bayer), Slack Publications (Chief Medical Editor, Cardiology Today's Intervention), Society of Cardiovascular Patient Care (Secretary/Treasurer), WebMD (CME steering committees); Other: Clinical Cardiology (Deputy Editor), NCDR-ACTION Registry Steering Committee (Chair), VA CART Research and Publications Committee (Chair); Research Funding: Abbott, Afimmune, Amarin, Amgen, AstraZeneca, Bayer, Boehringer Ingelheim, Bristol-Myers Squibb, Cardax, CellProthera, Cereno Scientific, Chiesi, CSL Behring, Eisai, Ethicon, Ferring Pharmaceuticals, Forest Laboratories, Fractyl, Garmin, HLS Therapeutics, Idorsia, Ironwood, Ischemix, Janssen, Lexicon, Lilly, Medtronic, MyoKardia, NirvaMed, Novartis, Novo Nordisk, Owkin, Pfizer, PhaseBio, PLx Pharma, Regeneron, Roche, Sanofi, Synaptic, The Medicines Company, 89Bio; Royalties: Elsevier (Editor, Cardiovascular Intervention: A Companion to Braunwald's Heart Disease); Site Co-Investigator: Abbott, Biotronik, Boston Scientific, CSI, St. Jude Medical (now Abbott), Philips, Svelte; Trustee: American College of Cardiology; Unfunded Research: FlowCo, Merck, Takeda. The other authors declare no competing interests.

\section{Peer review information}

Nature Reviews Cardiology thanks Steffen Massberg, Richard Vander Heide and the other, anonymous, reviewer(s) for their contribution to the peer review of this work.

\section{Publisher's note}

Springer Nature remains neutral with regard to jurisdictional claims in published maps and institutional affiliations.

C Springer Nature Limited 2021, corrected publication 202 